\title{
Dual droplet functionality: Phototaxis and
}

\section{Photopolymerization}

Sara Zarghami, ${ }^{\dagger, t}$ Yang Xiao, ${ }^{\dagger,+}$ Pawel Wagner, ${ }^{\dagger,+}$ Larisa Florea, ${ }^{\S}$ Dermot Diamond, " David L. Officer $^{t, t *}$ and Klaudia Wagner, ${ }^{t, *} *$

${ }^{\dagger}$ ARC Centre of Excellence for Electromaterials Science and $¥$ Intelligent Polymer Research Institute, AIIM Faculty, Innovation Campus, University of Wollongong, North Wollongong, NSW 2522, Australia.

${ }^{\S}$ Advanced Materials and BioEngineering Research (AMBER), Centre for Research on Adaptive Nanostructures and Nanodevices and School of Chemistry, Trinity College Dublin, Ireland.

"Insight Centre for Data Analytics, National Centre for Sensor Research, Dublin City University, Dublin 9, Ireland.

KEYWORDS: photopolymerization, phototaxis, photoactive microdroplets, chemical transport, Marangoni effect.

ABSTRACT: The use of phototaxis to move droplets in liquids offers the opportunity to emulate natural processes such as the controlled transport of materials in fluidic environments and to undertake chemistry at specific locations. We have developed a photoactive organic droplet whose movement in aqueous solution is driven by a photoinitiator, as a result of a light induced reaction within the droplet generating a Marangoni flow. The photoinitiator not only drives the droplet motion but can also be used to initiate polymerization following transfer of the droplet to a specific location and its merging 
with a secondary, monomer-containing droplet. The same light is used to control the transport of the droplet and the polymerization. The efficacy of this droplet transport and reactor system has been demonstrated by the site specific underwater polymerization of $\mathrm{N}$-isopropylacrylamide to repair a leaking vessel and the adhesion of two materials together.

\section{Introduction}

Remote activation of a reaction such as polymerization at a specific location would be very valuable, particularly in challenging environments such as under water. For example, defects in fluidic channels or tubes could be remotely repaired by directing each reactant of an adhesive to the defect and effecting the reaction at the point-of-need. Although this can be achieved by physical placement of the reactants using devices such as syringes or pipettes, the directed transport of individual reactants is a much more intriguing approach. One way to achieve this is through the controlled movement of reactant-containing droplets to locations of interest, where the polymerization reaction then occurs.

Droplet movement can be invoked and controlled using a variety of stimuli, such as chemical, thermal, and light amongst others.(1) Often, the droplet movement happens because of a chemical gradient in the external environment as demonstrated by Lagzi et al. who reported complex maze-solving behavior at the water-air interface using a simple fatty acid-containing organic liquid droplet propelled by $\mathrm{pH}$ regulated surface tension changes.(2) Control of the droplet movement was achieved through a combination of the $\mathrm{pH}$ of the aqueous medium and the confines of the maze channel geometry. Toyota and co-workers showed that an oil droplet containing a hydrolysis catalyst and hydrolyzable surfactant as fuel could be self-propelled at moderate speeds $\left(3-40 \mu \mathrm{m} \mathrm{s}^{-1}\right)$ on the surface of an aqueous medium.(3) Nonetheless, this movement still required a specific external environment (basic solution) and there was no control over the direction of motion. 
The manipulation of droplets with light has always been appealing and has been used to move oil droplets in water or water droplets in oil.(4) This has been achieved by both thermocapillary effects and chromocapillary effects, which result in droplet motion by changes in surface tension or Marangoni effects.(1,4) However, complete control of the movement of oil droplets both on and under water has only been recently demonstrated.(5) Xiao et al. developed an internal chromocapillary droplet motion in which a photoactive spiropyran or its merocyanine photoisomer present in an organic droplet could induce droplet movement in aqueous surfactant solution (Figure 1a) as a result of the spiropyran/merocyanine photoisomerization reaction changing the surface tension of the droplet/water interface (Figure 1b).(5) These droplets can carry a "reactant cargo", which can be delivered to and merged with a second droplet containing another reactant to perform a chemical reaction. This system offered the potential to achieve droplet-contained chemistry at a specific site, which could be useful for a variety of applications such as sensing, signaling, or material repair.

(a)

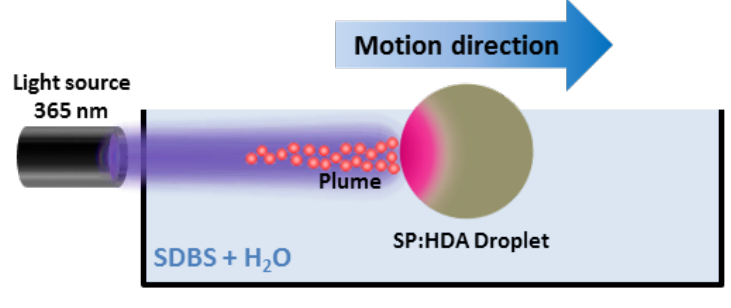

(b)

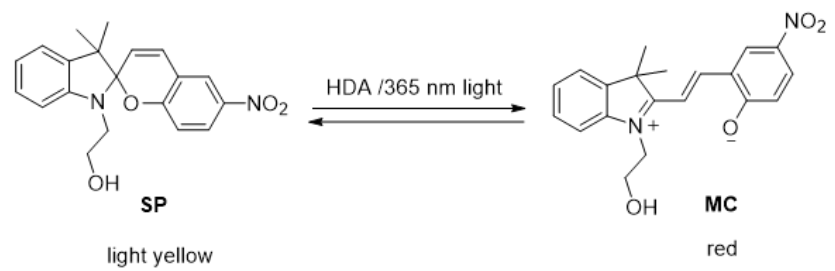

Figure 1. (a) Schematic representation of the directional movement away from the light source of a light-irradiated $(365 \mathrm{~nm})$ droplet containing nitrospiropyran (SP) and HDA. (b) Photoisomerization reaction of SP to nitromerocyanine (MC) in the presence of HDA that occurs in the droplet. Part (a) is reproduced with permission.(5) Copyright 2018 Wiley-VCH Verlag GmbH \& Co., KGaA, Weinheim. Inspired by recent developments in the use of synthetic polymers as wet adhesive materials, $(6-9)$ we have investigated site-specific photopolymerization via a droplet-based approach that can be achieved under water. Using our previous photoactive droplet system containing a commercially available nitro- 
substituted spiropyran (SP) and 2-hexyldecanoic acid (HDA) (Figure 1),(5) we have now demonstrated droplet-based transport of a photoinitiator to, and mixing with, a $N$-isopropylacrylamide (NIPAM) monomer droplet, which results in polymerization at a specific location under water. This has led to the discovery that the photoinitiator itself, phenylbis(2,4,6-trimethylbenzoyl)phosphine oxide (BAPO), can be used to induce both droplet movement and photopolymerization. In order to demonstrate possible applications of this droplet-based system, we have also shown that the BAPO-driven droplet system can be used to achieve polymerization at a specific location by blocking a water leak and bonding a Gortex strip to glass under water.

\section{Experimental Methods}

\section{Droplet Experiments}

Droplets were studied in glass Petri dishes (55 $\mathrm{mm}$ diameter) partially filled with an aqueous solution of sodium dodecylbenzene-sulfonate (SDBS) $(7 \mathrm{mM})$, a concentration well above its critical micelle

concentration $\left(\approx 1.5 \times 10^{-3} \mathrm{M}\right) .(10)$ The droplet speed was determined from movies, by calculating the time droplets took to cover specific distances.

\section{Movement of SP/HDA/DMPA Droplet 1A}

SP, HDA (1:1, mol/mol), and 2,2-dimethoxy-2-phenylacetophenone (DMPA) were dissolved in dichloroethane (DCE) to give concentrations of 0.1 and 4.5 M, respectively. An organic droplet was generated by loading $1 \mu \mathrm{L}$ of the above solution, using an autopipette, onto the bottom of the Petri dish containing the SDBS solution. On irradiation of the droplet with $365 \mathrm{~nm}$ light, it moved $8 \mathrm{~mm}$ away from the light with a speed of $0.5 \mathrm{~mm} \mathrm{~s}^{-1}$. 


\section{NIPAM/SDBS Droplet 2}

NIPAM $(0.1 \mathrm{~g})$ and SDBS $(1.7 \mathrm{mg})$ were dissolved in $50 \mu \mathrm{L}$ of DCE. An organic droplet was generated by loading $1 \mu \mathrm{L}$ of the above solution, using an autopipette, onto the bottom of the Petri dish containing the SDBS solution.

\section{Polymerization Using SP/HDA/DMPA Droplet 1A and NIPAM/SDBS Droplet 2 (System A)}

To achieve polymerization in system A, by using an autopipette, $1 \mu \mathrm{L}$ of droplet $1 \mathrm{~A}$ and $1 \mu \mathrm{L}$ of droplet 2 were formed on the bottom of the Petri dish containing the SDBS solution. Upon irradiation of droplet 1A with $365 \mathrm{~nm}$ light, it moved away from the light toward the immobile droplet 2 , whereupon the droplets collided and merged, initiating the polymerization reaction, which was essentially complete after almost 2 min of photoillumination as can be seen in Movie S1.

\section{SP/HDA/BAPO Droplet 1B}

SP, HDA (1:1, mol/mol), and BAPO were dissolved in DCE to give concentrations of 0.1 and $0.5 \mathrm{M}$, respectively. An organic droplet was generated by loading $1 \mu \mathrm{L}$ of the above solution, using an autopipette, onto the bottom of the Petri dish containing the SDBS solution. On irradiation of the droplet with $365 \mathrm{~nm}$ light, it moved $220 \mathrm{~mm}$ away from the light with a speed of $4.0 \mathrm{~mm} \mathrm{~s}^{-1}$ as can be seen in Movie S2.

\section{Polymerization Using SP/HDA/BAPO Droplet 1B and NIPAM/SDBS Droplet 2 (System B)}

To achieve polymerization in system $\mathrm{B}, 1 \mu \mathrm{L}$ of droplet $1 \mathrm{~B}$ and $1 \mu \mathrm{L}$ of droplet 2 were loaded onto the bottom of the Petri dish containing the SDBS solution using an autopipette. On irradiation of droplet 1B with $365 \mathrm{~nm}$ light, it moved away from the light toward droplet 2, whereupon the droplets collided 
and merged, initiating the polymerization reaction and forming a polymer after $40 \mathrm{~s}$ of photoillumination as can be seen in Movie S3.

\section{BAPO Droplet 1C}

BAPO was dissolved in DCE to give a concentration of $0.5 \mathrm{M}$. An organic droplet was generated by loading $1 \mu \mathrm{L}$ of the above solution, using an autopipette, onto the bottom of the Petri dish containing the SDBS solution. On irradiation of the droplet with $365 \mathrm{~nm}$ light, it moved $90 \mathrm{~mm}$ away from the light with a speed of $1.4 \mathrm{~mm} \mathrm{~s}^{-1}$ as can be seen in Movie S4.

\section{Polymerization Using BAPO Droplet 1C and NIPAM/SDBS Droplet 2 (System C)}

To achieve polymerization in system $\mathrm{C}, 1 \mu \mathrm{L}$ of droplet $1 \mathrm{C}$ and $1 \mu \mathrm{L}$ of droplet 2 were loaded onto the bottom of the Petri dish containing the SDBS solution using an autopipette. On irradiation of the droplet 1C with $365 \mathrm{~nm}$ light, it moved away from the light toward droplet 2, whereupon they collided and merged, forming a polymer after $40 \mathrm{~s}$ of photoillumination as can be seen in Movie S8.

\section{Leak Repair by Droplet Polymerization}

A hole was drilled in a glass Petri dish, which was then filled with aqueous SDBS solution. A NIPAM droplet (droplet 2) was placed as close as possible to the hole and the BAPO droplet (droplet 1C) was placed $1 \mathrm{~cm}$ from droplet 2. Droplet $1 \mathrm{C}$ was irradiated with $365 \mathrm{~nm}$ light and moved toward and merged with monomer droplet 2. The merged droplets moved to the hole upon continued irradiation. Further irradiation of the mixed droplets led to polymerization and sealing of the hole as can be seen in Movie S9. 


\section{Underwater Adhesion of Gortex to Glass by Droplet Polymerization}

A glass Petri dish was filled with aqueous SDBS and a small glass slide $(0.4 \mathrm{~g})$ was placed on the bottom. A strip of hydrophobic PTFE Teflon-based membrane (Gortex) $(3 \times 1 \mathrm{~cm})$ was immersed into the solution in contact with the glass slide. NIPAM droplet $2(20 \mu \mathrm{L})$ was placed underwater at the base of the Gortex membrane and $3 \mu \mathrm{L}$ of BAPO droplet $1 \mathrm{C}$ was placed on the glass slide. The BAPO droplet was irradiated with $365 \mathrm{~nm}$ light and directed to merge with the NIPAM droplet on the Gortex strip. Continued irradiation for $4 \mathrm{~min}$ led to polymerization of the NIPAM and attachment of the Gortex membrane to the glass slide as can be seen in Movie S10.

\section{Results and Discussion}

Initially, the ability of the SP/HDA droplet system to transport a photoinitiator was investigated. A neutral organic droplet $(1 \mu \mathrm{L})$ comprising SP/HDA (1:1) and DMPA (Figure 2a) in DCE was prepared. DMPA was chosen as the photoinitiator as it is commonly used for the free radical polymerization of NIPAM (see Figure S1a).(11,12) The droplet was placed under an aqueous SDBS solution and irradiated with a $365 \mathrm{~nm}$ light source, which led to droplet movement away from light, consistent with the SP/HDA droplet previously reported (Figure 1a).(5) However, the movement was substantially limited with the droplet only traveling $8 \mathrm{~mm}$ at a speed of $0.5 \mathrm{~mm} \mathrm{~s} \mathrm{~s}^{-1}$. As previously

reported,(5) SP/HDA droplets can move over several centimeters at speeds of up to $1.2 \mathrm{~mm} \mathrm{~s}^{-1}$. The reduced speed and lifetime of the DMPA-containing droplet suggested that the photoinitiator had inhibited the droplet motion. 
(a)<smiles>C/C=C\C(=O)NC(C)C</smiles>

NIPAM (Monomer)

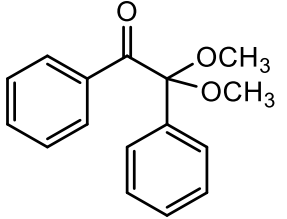

DMPA (Initiator)

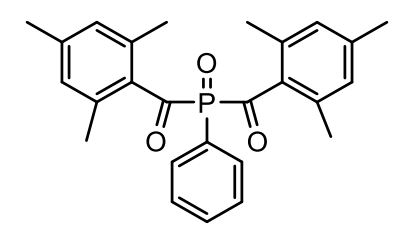

BAPO (Initiator)

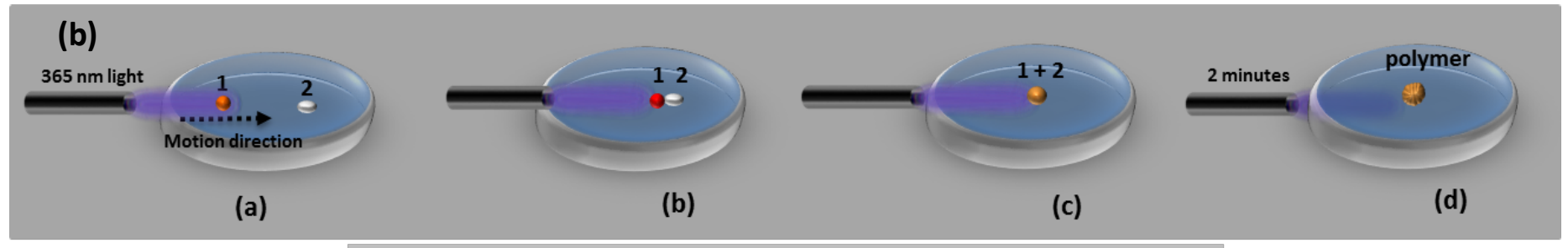

System A: Droplet 1A (SP, HDA, DMPA), D Droplet 2 (NIPAM, SDBS)

System B: - Droplet 1B (SP, HDA, BAPO), D Droplet 2 (NIPAM, SDBS)

System C: Droplet 1C (BAPO), - Droplet 2 (NIPAM, SDBS)

Figure 2. (a) Structures of the NIPAM monomer and the photoinitiators (DMPA and BAPO). (b) Schematic representation of the movement of the photoactive droplet 1 to mix with droplet 2 to achieve polymerization and the description of the droplets used in each system.

Nonetheless, this DMPA-containing droplet was used to investigate the localized polymerization of NIPAM (Figure 2a) as polymers of NIPAM have been used in several applications in microfluidics such as for flow controllers and actuators, $(13-15)$ drug delivery systems, and bioassays.(16,17) The DMPA droplet (droplet 1A) was placed under the surfactant solution in a glass Petri dish close to $1 \mu \mathrm{L}$ of a second DCE droplet (droplet 2) containing NIPAM and the anionic surfactant, SDBS (Figure 2b, system A). SDBS was added to droplet 2 to improve the mixing of the two droplets. As shown in Movie S1 and Figure 2b, droplet 1A could be guided using the $365 \mathrm{~nm}$ light to droplet 2 and merged with it, resulting in the formation of an orange solid polymer after irradiating the droplet mixture for a further $2 \mathrm{~min}$. The color of the polymer pNIPAM arises from the presence of the highly colored SP photoisomer, nitromerocyanine (MC, Figure 1b), which is formed during illumination. 
To increase the efficiency of polymerization, we changed the DMPA photoinitiator to BAPO (Figure 2a).(18,19)Given that the BAPO absorption spectrum (Figure S2b) contained a more significant absorption at $365 \mathrm{~nm}$ than DMPA (Figure S1b), it was likely that more organic free radicals could be generated to increase the rate of polymerization.(20) Irradiation $(365 \mathrm{~nm})$ of a DCE droplet $(1 \mu \mathrm{L})$ containing SP/HDA/BAPO $(1 \mu \mathrm{L})$ resulted in droplet movement away from the light (Movie S2) as it occurred previously for DMPA-containing droplets, but the droplet speed was much higher $(4.0 \mathrm{~mm} \mathrm{~s}$ $\left.{ }^{1}\right)$ compared to the speed of the DMPA system A $\left(0.5 \mathrm{~mm} \mathrm{~s}^{-1}\right)$ droplet and the SP/HDA droplet without any photoinitiator $\left(1.2 \mathrm{~mm} \mathrm{~s}^{-1}\right) .(5)$ In addition, the droplet motion was more erratic in its direction than that observed with a SP/HDA droplet. As it has been demonstrated that the movement of these types of photoactive droplets is driven by a change in interfacial tension (IFT) and resulting Marangoni flows,(5) the higher speed and erratic movement of the SP/HDA/BAPO droplet suggested that the light-induced fragmentation of BAPO itself (Figure S2a) could also induce a differential IFT, contributing to an overall increase in the IFT gradient.(21)

Polymerization using this SP/HDA/BAPO droplet was further investigated. Droplet 1B contained SP/HDA/BAPO in DCE $(1 \mu \mathrm{L})$, whereas the stationary droplet 2 comprised NIPAM/SDBS (Figure $2 \mathrm{~b}$, system B). Irradiation of droplet 1B with $365 \mathrm{~nm}$ light in an aqueous SDBS solution led to rapid merging of the two droplets with a faster polymerization rate, taking less than $1 \mathrm{~min}$ to form the reddish solid polymer (Movie S3 and Figure 2b).

Given the apparent effect of the BAPO on the droplet speed, we investigated whether BAPO itself could induce droplet movement. A $1 \mu \mathrm{L}$ DCE droplet containing only BAPO $(0.5 \mathrm{M})$ was irradiated in an aqueous SDBS solution with $365 \mathrm{~nm}$ light and the droplet did indeed move away from the light as shown in Movie S4. This droplet traveled $90 \mathrm{~mm}$ with a speed of $1.4 \mathrm{~mm} \mathrm{~s}^{-1}$. Varying the concentration of BAPO showed that the speed could be increased to $2.0 \mathrm{~mm} \mathrm{~s}^{-1}$ for a $2 \mathrm{M}$ BAPO 
droplet, which traveled $390 \mathrm{~mm}$ (Table S1). This was the maximum amount of BAPO that could be dissolved in DCE. No significant movement was observed for droplets containing concentrations of BAPO below $0.25 \mathrm{M}$. In addition, the distance traveled could be increased with the size of the droplet (Table S2), up to $1120 \mathrm{~mm}$ for a $15 \mu \mathrm{L}$ BAPO droplet. Droplets larger than $15 \mathrm{~mL}$ tended to flatten and became difficult to move.

We also examined the movement of the DMPA droplet, but it barely moved, most likely because of the insufficient overlap of its absorbance spectrum with the $365 \mathrm{~nm}$ light source. At a DMPA concentration of $0.5 \mathrm{M}$ (the same concentration used in the BAPO droplet) the speed obtained was $0.11 \mathrm{~mm} \mathrm{~s}^{-1}$, ceasing after only $4 \mathrm{~mm}$ of movement. The DMPA concentration was increased to $4.5 \mathrm{M}$ (the concentration used for polymerization) and the speed and distance improved marginally to $0.35 \mathrm{~mm} \mathrm{~s}^{-}$ ${ }^{1}$ and $10 \mathrm{~mm}$, respectively.

To investigate the BAPO droplet movement further, the IFT was measured using a BAPO/DCE pendant droplet suspended in DI water before and after irradiation (Figure S3), although this could only be carried out in water without the presence of a surfactant. Irradiation of the BAPO droplet ( $365 \mathrm{~nm})$ led to a $\sim 5 \mathrm{mN} \mathrm{m}^{-1}$ decrease in IFT, consistent with that previously observed for SP.(5) This results from photocleavage of BAPO (Figure S2a) and presumably subsequent reaction of the resulting radicals with a radical-scavenging species like oxygen.(19)

As previously reported for self-propelled droplets and spiropyran photoactive droplets, $(5,21)$ droplet movement can be explained if a species within a microdroplet undergoes a chemical interaction with the surfactant molecules, generating an internal Marangoni convection flow on the droplet surface, resulting in an external Marangoni flow. An internal current can be seen in an irradiated immobile BAPO droplet (Figure 3, Movie S5). The introduction of 10-25 $\mu \mathrm{m}$ glass microbeads into the immobilized droplet, which moved to the liquid interface as a result of their hydrophilicity, allowed the 
effective visualization of the external Marangoni current as can be seen in Movie S6. This Marangoni flow was further confirmed by the movement of carbon particles in the surrounding aqueous medium across the surface of the moving droplet (Movie S7).

(a)
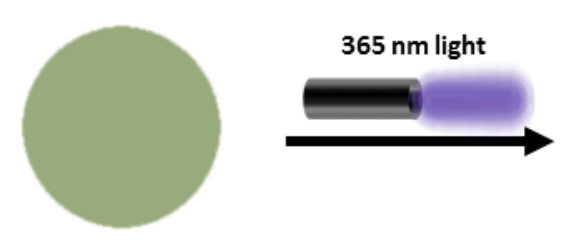

(b)
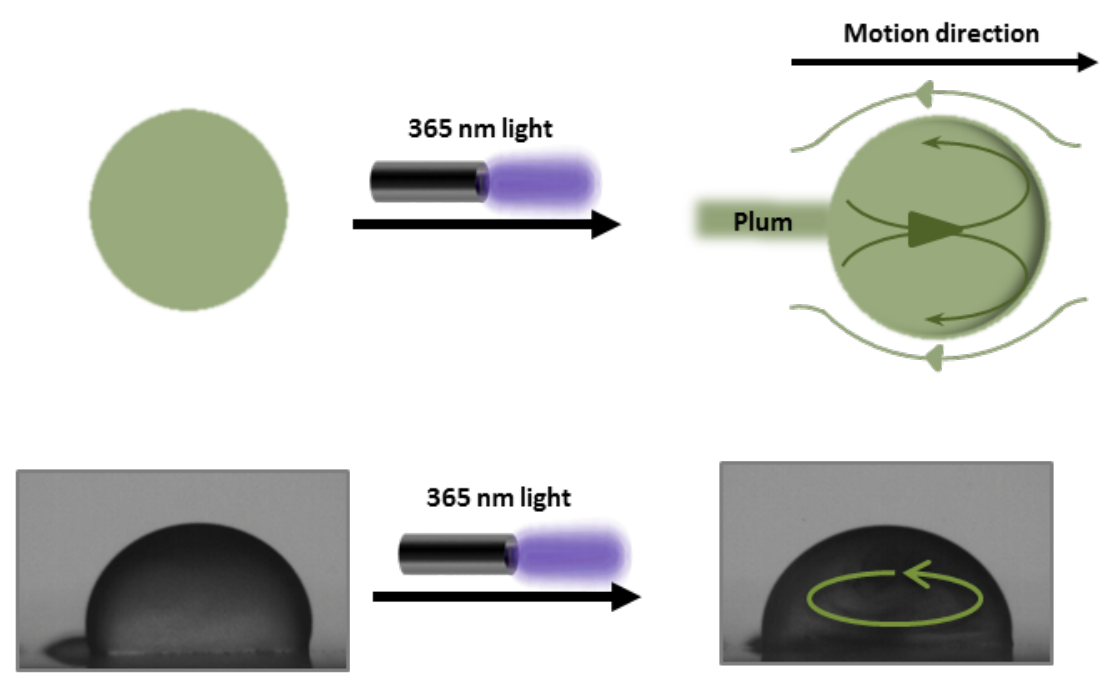

Figure 3. (a) Schematic representation of BAPO droplet 1C moving by Marangoni flow. (b) Side view of Marangoni flow in the droplet (from Movie S5).

Therefore, it is likely that the photocleavage of BAPO leads to a change in surfactant distribution on the surface of the droplet as a result of the interaction of the photocleavage product with the surfactant inducing an internal droplet fluid flow and creating an IFT gradient that is continuously sustained. This leads to the external Marangoni flow in the direction of the higher IFT that results in mass transfer along the liquid interface (Marangoni effect),(21-23) propelling the droplet away from the light source, as previously shown for other droplets moving by an analogous chromocapillary effect.(5,24)

While the droplet was moving, a narrow plume emerging from the droplet was visible (Movie S4). This is not surprising given previous observations of plumes from photoactivated droplets and the reported advection of materials from droplets driven by chemical reactions.(5) To attempt to identify the 
components of this plume, UV-vis spectra were collected from an irradiated $(365 \mathrm{~nm})$ pendant BAPO droplet immersed in water (see Figure S4a). The spectra of the plume (Figure S4b) with the absorptions at 360 and $280 \mathrm{~nm}$ suggested that BAPO was present in the released material. However, other aromatic components from the BAPO photodegradation may have also contributed to the observed absorption.

The photoinitiator droplet movement provided the opportunity to use this simpler system without SP for polymerization. Therefore, droplet 1C (BAPO) was illuminated with $365 \mathrm{~nm}$ light, whereupon it moved toward and successfully merged with the NIPAM-containing droplet 2 (Movie $\underline{\mathrm{S} 8}$ and Figure $2 \mathrm{~b}$, system C). The resulting polymer was obtained after only $40 \mathrm{~s}$ of further light irradiation. The completion of the polymerization was determined by infrared spectroscopy (Figure S5), which showed an absence of the NIPAM monosubstituted $\mathrm{C}=\mathrm{C}$ (vinyl) stretch at $991 \mathrm{~cm}^{-1}$, with the spectrum consistent with that of pNIPAM as previously reported.(25)

The movement of a photoinitiator-based droplet provided the opportunity to carry out proof-of-concept polymerizations at defined locations at which monomer droplets were placed. The repair of a leaking vessel was initially undertaken to demonstrate this. A hole was created in a Petri dish that was then filled with aqueous SDBS solution. A NIPAM monomer droplet was placed close to the hole and a BAPO droplet positioned $1 \mathrm{~cm}$ away as shown in Figure $4 \mathrm{a}(\mathrm{i})$. The irradiated BAPO droplet $1 \mathrm{C}$ moved toward and merged with the NIPAM droplet. The merged droplets continued to move to cover the hole and, under further illumination, polymerization occurred, completely sealing the hole and stopping the water leak (Figure 4a(ii-iv), Movie S9). 
(a)

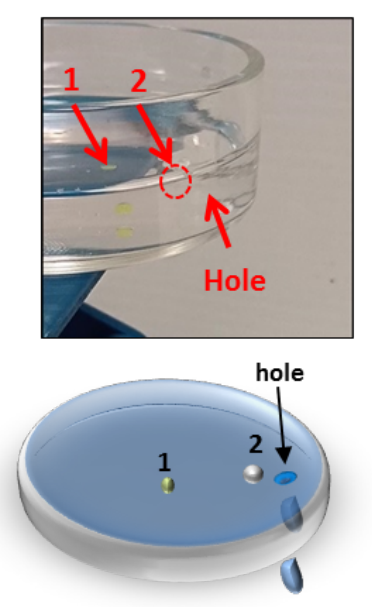

(i)

(b)
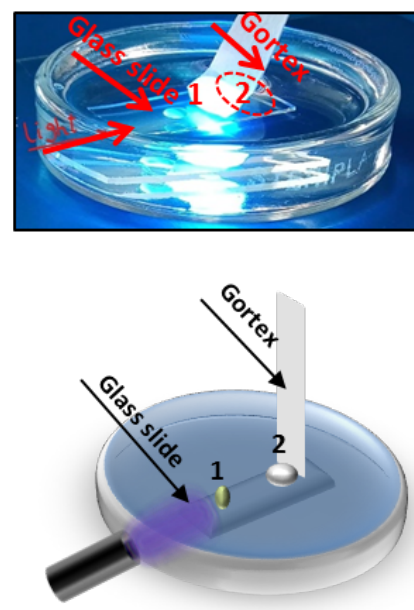

(i)
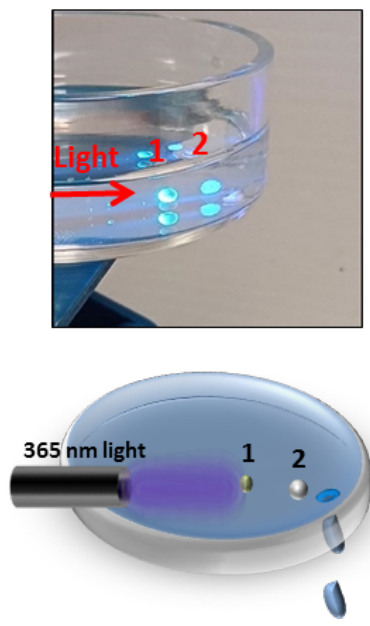

(ii)
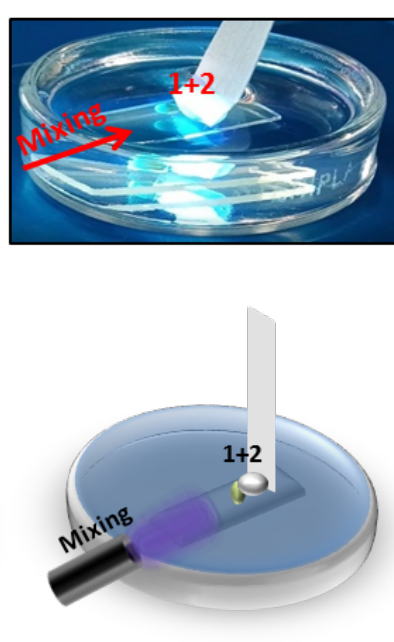

(ii)
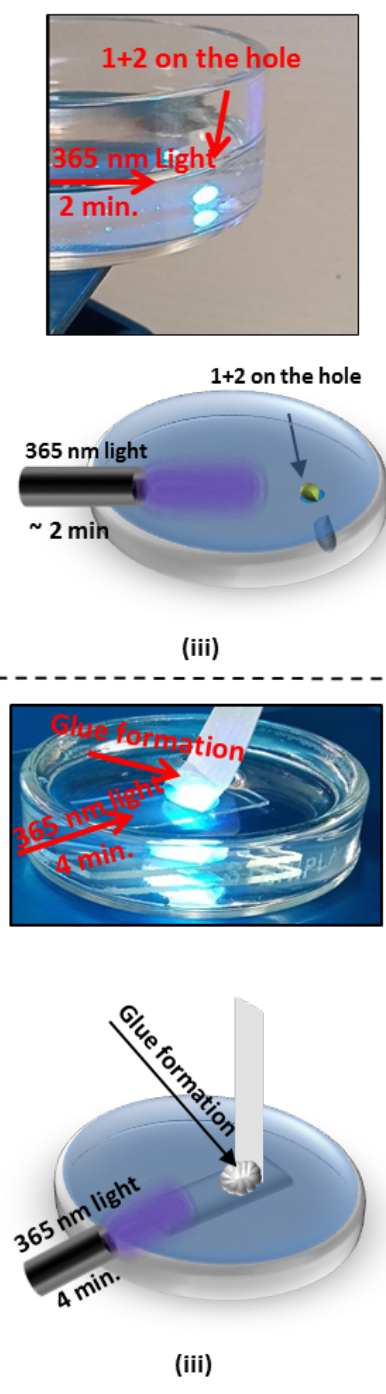
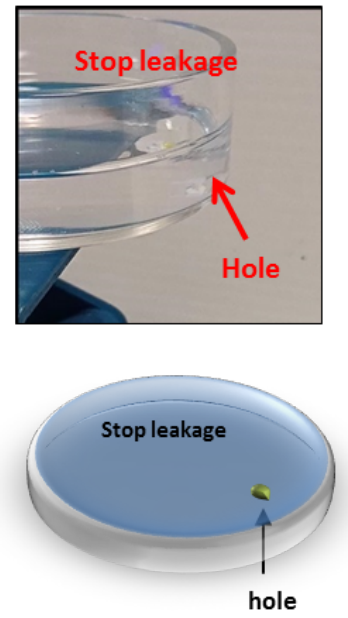

(iv)
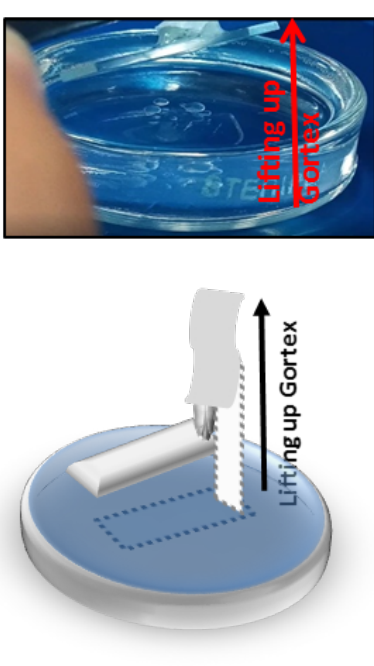

(iv)

Figure 4. Schematic representation of (a) blocking a hole to stop water leakage and (b) underwater adhesion of a Gortex strip to glass. Photographs and illustrations of (i) initial droplet arrangement, (ii) light-activated movement and mixing of the droplets, (iii) droplet photopolymerizations, (iv) demonstration of polymerization outcomes for both (a) hole blocking and (b) Gortex strip attachment. In both (a,b), all photographs were taken from $\underline{\text { Movies S9 }}$ and $\underline{\mathrm{S} 10}$, respectively.

A further qualitative demonstration of the use of this polymerization system was undertaken by adhering two different surfaces together under water. A glass Petri dish was filled with aqueous SDBS solution and a small glass slide $(0.4 \mathrm{~g})$ was placed on the bottom of the Petri dish. A strip of 
hydrophobic PTFE-based membrane (Gortex) was immersed into the solution in contact with the glass slide. Following that, a $20 \mu \mathrm{L}$ monomer droplet 2 (NIPAM/SDBS/DCE) was placed on the edge of the Gortex membrane and a $3 \mu \mathrm{L}$ droplet of BAPO (droplet 1C) placed on the glass slide (Figure $4 \mathrm{~b}(\mathrm{i})$ ). The BAPO droplet was then driven with light toward the Gortex strip and merged with the monomer droplet 2 (Figure $4 \mathrm{~b}(\mathrm{ii})$ ). Irradiation of the mixed droplets for a further 4 min led to polymerization of the NIPAM (Figure $4 \mathrm{~b}(\mathrm{iii})$ ) and attachment of the Gortex membrane to the glass slide as can be seen in Figure 4b(iv) and Movie S10.

\section{Conclusions}

In summary, we have demonstrated that photoactive droplets containing a nitrospiropyran (SP) can be moved using light and used to transport polymerization photoinitiators to a second monomercontaining droplet, leading to photopolymerization at a specific location. Thus, whereas the photoactive droplet system containing SP and surfactant HDA in DCE was shown to move photoinitiator DMPA up to $8 \mathrm{~mm}$ at a speed of $0.5 \mathrm{~mm} \mathrm{~s}^{-1}$, the analogous SP/HDA/BAPO droplet was still moving after $60 \mathrm{~mm}$ at $4.0 \mathrm{~mm} \mathrm{~s}^{-1}$. In both cases, the movement was somewhat erratic compared to the droplet without a photoinitiator, suggesting that decomposition of the photoinitiator itself was playing a role in droplet movement. This led to the discovery that the photoinitiators could be used to move the organic droplets without the need for SP.

BAPO was found to be particularly effective both at producing photogenerated movement because of its efficient light absorption at $365 \mathrm{~nm}$ and faster photopolymerization. Depending on their concentration and size, BAPO/DCE droplets traveled up to $1120 \mathrm{~mm}$ and speeds of $2.1 \mathrm{~mm} \mathrm{~s}^{-1}$. A BAPO droplet could be merged with a NIPAM droplet to effect photopolymerization in half the time compared to a SP/HDA/DMPA droplet. IFT studies into the mechanism of BAPO droplet movement 
suggested that photofragmentation of BAPO caused a similar decrease in IFT to that obtained during photoisomerization of the SP photoswitch as previously demonstrated for other droplets driven by the chromocapillary effect.

The BAPO droplet was then used to demonstrate its potential for remote controlled polymerization at specific locations under water. A hole in a Petri dish was able to be sealed using merged BAPO and NIPAM droplets and the same approach was used to adhere two different surfaces together under water. This ability to direct movement of reactant-containing droplets to predetermined locations using light whereupon advanced functions like fluidic leak repair can be performed demonstrates one of many potential possibilities of using this type of site-directed chemistry in futuristic microfluidic systems. (26)

\section{Supporting Information}

The Supporting Information is available free of charge on the ACS Publications website at DOI: $\underline{10.1021 / \text { acsami.9b08697. }}$

- Additional experimental details including materials, instrumentation and characterization, methods, details of DMPA, details of BAPO, IFT measured by the pendant drop method, experimental setup and UV/vis spectra of BAPO droplet plume, FTIR spectra of NIPAM and pNIPAM, effect of BAPO concentration, and effect of size of a 2M BAPO droplet (PDF)

- Movement of droplet 1A (SP:HDA:DMPA) and mixing with droplet 2 (NIPAM:SDBS) (MP4)

- $\quad$ Movement of droplet 1B (SP:HDA:BAPO) (MP4)

- Movement of droplet 1B (SP:HDA:BAPO) and mixing with droplet 2 (NIPAM:SDBS) (MP4)

- Movement of a BAPO droplet (MP4)

- Internal Marangoni convection current with a stationary $3 \mu \mathrm{L}$ BAPO droplet (MP4) 
- Marangoni current on the surface of a $1 \mu \mathrm{L}$ BAPO droplet containing 10-25 $\mu \mathrm{m}$ glass microbeads (MP4)

- Marangoni flow around a BAPO droplet in a carbon nanopowder-containing aqueous medium (MP4)

- Movement of droplet 1C (BAPO) and mixing with droplet 2 (NIPAM:SDBS) (MP4)

- Use of a system C droplet to repair a hole and stop water leakage (MP4)

- Use of a system C droplet to adhere glass and Gortex surfaces together under water (MP4)

Author Contributions

The paper was written through contributions of all the authors. All the authors have given approval to the final version of the paper.

The authors declare no competing financial interest.

\section{Acknowledgments}

This work was supported by the Australian Research Council (ARC) Discovery Project DP 150104532 and the ARC Centre of Excellence Scheme (project no. CE 140100012). The authors also acknowledge support from Science Foundation Ireland under the Insight Centre (grant no. SFI/12/RC/2289). L.F. acknowledges the ERC (European Research Council) Starting Grant (project number 802929ChemLife). The authors thank the Australian National Fabrication Facility for access to specialized facilities. 


\section{References}

1. Lach, S.; Yoon, S. M.; Grzybowski, B. A. Tactic, Reactive, and Functional Droplets Outside of Equilibrium. Chem. Soc. Rev. 2016, 45,4766- 4796, DOI: 10.1039/c6cs00242k

2. Lagzi, I.; Soh, S.; Wesson, P. J.; Browne, K. P.; Grzybowski, B. A. Maze Solving by Chemotactic Droplets. J. Am. Chem. Soc. 2010, 132,1198-1199, DOI: 10.1021/ja9076793

3. Toyota, T.; Maru, N.; Hanczyc, M. M.; Ikegami, T.; Sugawara, T. Self-Propelled Oil Droplets Consuming "Fuel" Surfactant. J. Am. Chem. Soc. 2009, 131, 5012- 5013, DOI: $10.1021 / \mathrm{ja} 806689 \mathrm{p}$

4. Baigl, D. Photo-Actuation of Liquids for Light-Driven Microfluidics: State of the Art and Perspectives. Lab Chip 2012, 12, 3637-3653,DOI: 10.1039/c2lc40596b

5. Xiao, Y.; Zarghami, S.; Wagner, K.; Wagner, P.; Gordon, K. C.; Florea, L.; Diamond, D.; Officer, D. L. Moving Droplets in 3D Using Light.Adv. Mater. 2018, 30, 1870259, DOI: 10.1002/adma.201870259

6. North, M. A.; Del Grosso, C. A.; Wilker, J. J. High Strength Underwater Bonding with Polymer Mimics of Mussel Adhesive Proteins.ACS Appl. Mater. Interfaces 2017, 9, 7866- 7872, DOI: 10.1021/acsami.7b00270

7. Zhao, Y.; Wu, Y.; Wang, L.; Zhang, M.; Chen, X.; Liu, M.; Fan, J.; Liu, J.; Zhou, F.; Wang, Z. Bio-Inspired Reversible Underwater Adhesive.Nat. Commun. 2017, 8, 2218, DOI: $10.1038 / \mathrm{s} 41467-017-02387-2$

8. Chipara, A. C.; Tsafack, T.; Owuor, P. S.; Yeon, J.; Junkermeier, C. E.; van Duin, A. C. T.; Bhowmick, S.; Asif, S. A. S.; Radhakrishnan, S.;Park, J. H.; Brunetto, G.; Kaipparettu, B. A.; Galvão, D. S.; Chipara, M.; Lou, J.; Tsang, H. H.; Dubey, M.; Vajtai, R.; Tiwary, C. 
S.; Ajayan, P. M.Underwater Adhesive Using Solid-Liquid Polymer Mixes. Mater. Today

Chem. 2018, 9, 149-157, DOI: 10.1016/j.mtchem.2018.07.002

9. Li, X.; Li, W.; Liu, Z.; Wang, X.; Guo, H.; Wang, R.; Guo, X.; Li, C.; Jia, X. Underwater Polyurethane Adhesive with Enhanced Cohesion by Postcrosslinking of Glycerol Monomethacrylate. J. Appl. Polym. Sci. 2018, 135, 46579, DOI: 10.1002/app.46579

10. Yu, D.; Huang, F.; Xu, H. Determination of Critical Concentrations by Synchronous Fluorescence Spectrometry. Anal. Methods2011, 4, 47-49, DOI: 10.1039/c1ay05495c

11. Uludag, H.; Wong, M.; Man, J. Reactivity of Temperature-Sensitive, Protein-Conjugating Polymers Prepared by a Photopolymerization Process. J. Appl. Polym. Sci. 2000, 75, 583592, DOI: 10.1002/(sici)1097-4628(20000124)75:4<583::aid-app15>3.0.co;2-1

12. Ziółkowski， B.; Ates， Z.; Gallagher， S.; Byrne， R.; Heise， A.; Fraser， K. J.; Diamond, D. Mechanical Properties and UV Curing Behavior of Poly(N-Isopropylacrylamide) in Phosphonium-Based Ionic Liquids. Macromol. Chem. Phys. 2013, 214, 787-796, DOI: 10.1002/macp.201200616

13. Dong, L.; Jiang, H. Autonomous Microfluidics With Stimuli-Responsive Hydrogels. Soft Matter 2007, 3, 1223-1230, DOI: 10.1039/b706563a

14. Richter, A.; Klatt, S.; Paschew, G.; Klenke, C. Micropumps Operated by Swelling and Shrinking of Temperature-Sensitive Hydrogels.Lab Chip 2009, 9, 613-618, DOI: $10.1039 / \mathrm{b} 810256 \mathrm{~b}$

15. Satoh, T.; Sumaru, K.; Takagi, T.; Kanamori, T. Fast-Reversible Light-Driven Hydrogels Consisting of Spirobenzopyran-Functionalized Poly(N-Isopropylacrylamide). Soft Matter 2011, 7, 8030- 8034, DOI: 10.1039/c1sm05797a 
16. Benito-Lopez, F.; Byrne, R.; Răduţă, A. M.; Vrana, N. E.; McGuinness, G.; Diamond, D. Ionogel-Based Light-Actuated Valves for Controlling Liquid Flow in Micro-Fluidic Manifolds. Lab Chip 2010, 10, 195-201, DOI: 10.1039/b914709h

17. Liu, F.; Urban, M. W. Recent Advances and Challenges in Designing Stimuli-Responsive Polymers. Prog. Polym. Sci. 2010, 35, 3-23, DOI: 10.1016/j.progpolymsci.2009.10.002

18. Kolczak, U.; Rist， G.; Dietliker， K.; Wirz， J. Reaction Mechanism of Monoacyl- and Bisacylphosphine Oxide Photoinitiators Studied by ${ }^{31} \mathrm{P}-,{ }^{13} \mathrm{C}-$, and ${ }^{1} \mathrm{H}-\mathrm{CIDNP}$ and ESR. J. Am. Chem. Soc. 1996, 118, 6477-6489, DOI: 10.1021/ja9534213

19. Jockusch, S.; Turro, N. J. Phosphinoyl Radicals: Structure and Reactivity. A Laser Flash Photolysis and Time-Resolved ESR Investigation. J. Am. Chem. Soc. 1998, 120,1177311777, DOI: $10.1021 / \mathrm{ja} 982463 z$

20. Meereis, C. T. W.; Leal, F. B.; Lima, G. S.; de Carvalho, R. V.; Piva, E.; Ogliari, F. A. BAPO as an Alternative Photoinitiator for the Radical Polymerization of Dental Resins. Dent. Mater. 2014, 30, 945-953, DOI: 10.1016/j.dental.2014.05.020

21. Maass, C. C.; Krüger, C.; Herminghaus, S.; Bahr, C. Swimming Droplets. Annu. Rev. Condens. Matter Phys. 2016, 7, 171- 193, DOI: 10.1146/annurev-conmatphys-031115-011517

22. Schmitt, M.; Stark, H. Marangoni Flow at Droplet Interfaces: Three-Dimensional Solution and Applications. Phys. Fluids 2016, 28,012106, DOI: 10.1063/1.4939212

23. Stricker, L. Numerical Simulation of Artificial Microswimmers Driven by Marangoni Flow. 2017, arXiv preprint arXiv:1702.03362.

24. Diguet, A.; Guillermic, R.-M.; Magome, N.; Saint-Jalmes, A.; Chen, Y.; Yoshikawa, K.; Baigl, D. Photomanipulation of a Droplet by the Chromocapillary Effect. Angew. Chem., Int. Ed. 2009, 48, 9281- 9284, DOI: 10.1002/anie.200904868 
25. Rwei, S.-P.; Chuang, Y.-Y.; Way, T.-F.; Chiang, W.-Y.; Hsu, S.-P. Preparation of Thermo- and pH-Responsive Star Copolymers Via ATRP and Its Use in Drug Release Application. Colloid Polym. Sci. 2015, 293, 493- 503, DOI: 10.1007/s00396-014-3436-0

26. ter Schiphorst, J.; Saez, J.; Diamond, D.; Benito-Lopez, F.; Schenning, A. P. H. J. LightResponsive Polymers for Microfluidic Applications. Lab Chip 2018, 18, 699-709, DOI: $10.1039 / \mathrm{c} 7 \mathrm{lc} 01297 \mathrm{~g}$ 